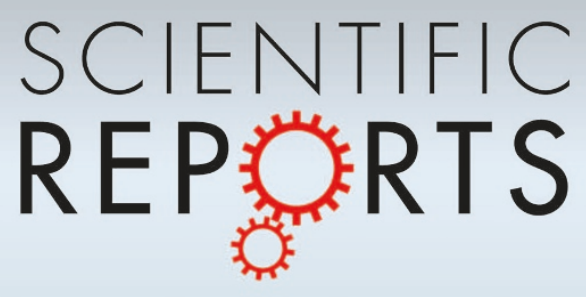

OPEN

SUBJECT AREAS:

NEUROSCIENCE

VISUAL SYSTEM

DEVELOPMENT OF THE NERVOUS

SYSTEM

PERCEPTION

Received

17 April 2013

Accepted

31 May 2013

Published

18 June 2013

Correspondence and requests for materials should be addressed to J.-W.Z. (jiawei.zhou@ mcgill.ca) or C.-B.H. (huangcb@psych.ac.

\section{The Effect of Unilateral Mean Luminance on Binocular Combination in normal and amblyopic vision}

\author{
Jiawei Zhou' ${ }^{1,2}$, Wuli Jia' ${ }^{1}$ Chang-Bing Huang' \& Robert F. Hess ${ }^{2}$
}

'Key Laboratory of Behavioral Science, Institute of Psychology, Chinese Academy of Sciences, Beijing, China, $100101,{ }^{2}$ McGill
Vision Research, Department of Ophthalmology, McGill University, Montreal, Quebec, Canada, H3A 1A1.

Luminance plays a modulating role in the processes of several visual tasks, which in turn provides significant information for the understanding of visual processing. Here, using a binocular phase combination paradigm, we studied the effect of unilateral changes in mean luminance on binocular combination in both normal and amblyopic vision. We found, in normal observers, attenuation of one eye's stimulus luminance with neutral density filters produces binocular phase combination similar to those of amblyopic subjects. Correspondingly, in amblyopic observers, reduction of the fellow eye's stimulus luminance produces binocular phase combination similar to those of normal subjects. These phenomena could be explained by an attenuated contribution of the filtered eye to the binocular phase percept due to reduced gain-control. The findings have major implications both for the study of binocular combination and for amblyopia treatment.

$\mathrm{N}$ umerous studies have shown that decreasing luminance in one eye can significantly affect subjects' monocular performance, such as visual acuity ${ }^{1,2}$, contrast sensitivity ${ }^{3-7}$ and visual resolution ${ }^{8}$ in the luminance-attenuated eye. These effects are scale-dependent ${ }^{5-7}$ and are understood within the framework of the quantal limitations ${ }^{9}$ and gain control of retinal cells with receptive fields of different sizes ${ }^{10}$. It has also been shown that decreasing the mean luminance in one eye can also affect binocular functions, e.g. stereoacuity $^{1,11}$, dichoptic contrast detection ${ }^{12-14}$, binocular space perception ${ }^{15}$, binocular rivalry ${ }^{16}$, binocular visual evoked potential ${ }^{17,18}$, and binocular sensory dominance ${ }^{19}$. These results indicate that an interocular luminance difference can induce a functional imbalance between the two eyes in normal observers, reducing binocular function, altering interocular interactions and shifting sensory dominance to the eye with the higher luminance level.

On the other hand, interocular differences in luminance can simulate the suppression-based imbalance in amblyopia ${ }^{19}$ and may restore balance (i.e., equal sensory dominance) between the two functionally imbalanced eyes in the case of amblyopia, For example, Leonards and Sireteanu ${ }^{16}$ reported that the abnormal time course of binocular rivalry could be "nomalized" by attenuating the mean luminance of the fellow eye's stimulus, and Hess and Mansouri et al. ${ }^{20}$ reported on the restoration of stereoscopic function in the eyes of some strabismic amblyopes by reducing the mean luminance of the dominant eye.

Although these previous studies clearly suggested that luminance may strongly modulate the functional balance between the two eyes in both normal and amblyopic observers, few studies have quantitatively determined the relationship between the interocular luminance difference and the degree of functional imbalance (i.e., the relative sensory dominance). With a dichoptic random dot motion paradigm, Zhang, Bobier, Thompson and Hess $^{19}$ evaluated the effect of mean luminance on the binocular sensory balance in five normal observers and found that the mean interocular contrast ratio corresponding to the binocular sensory balance status decreased from 0.88 , when the two eyes had same luminance, to around 0.1 , when the luminance in non-dominant eye was attenuated by a factor of 1000 . In the present study, we investigated the role of interocular luminance difference on supra-threshold binocular phase combination, from which we can derive a functional balance index between the two eyes, in both normals and amblyopes. We also aimed to determine whether one can artificially simulate "amblyopic" behaviour in normal observers and recover "normal" response patterns in amblyopic observers by inducing luminance differences in the two eyes. Finally, we sought to understand the physiological basis of such effects. 
The binocular phase combination paradigm was first developed by Ding and Sperling ${ }^{21}$, in which two monocular sine-wave gratings with varied contrast and spatial phase (shifted in opposite directions but with the same magnitude) are dichoptically presented to two eyes. The contrast of the sine-wave grating is fixed in one eye and increases proportionally in the other eye. The perceived phase of the cyclopean grating is determined by the internal representations of the two monocular gratings. It will monotonically shift from the phase of the grating in the contrast-fixed eye to that in the contrast-varied eye as the interocular contrast ratio increased from 0 to 1 , indicating a sensory dominance departure from the contrastfixed eye. The zero-crossing point of the perceived phases versus interocular contrast ratio (PvR) curve, also called the effective contrast ratio at balance point ${ }^{22-24}$, represents a measure of when the internal representations of two eyes' inputs are equal in binocular combination. In the current study, we measured the PvR curves with and without neutral density (ND) filters in both normal and amblyopic observers. An illustration of the experimental design is shown in Figure 1. We kept the contrast of the grating fixed in the left eye of normal observers, and found their PvR curves tend to demonstrate 'amblyopic' fashion when the mean luminance in this eye was reduced by an ND filter. Similarly, we kept the contrast of the grating in the amblyopic eye fixed in amblyopic observers, and found their PvR curves tend to show 'normal' properties when the mean luminance in the fellow eye was reduced by an ND filter. These results can be well explained by a modified contrast gain-control model ${ }^{21}$, which suggests that the decreasing of mean luminance in one eye reduces the efficiency of gain-control from that eye to the other eye, thus reducing its contribution in binocular combination.

\section{Results}

Normal subjects. The perceived phases versus interocular contrast ratio (PvR) curves at four neutral density filter transmission levels for three normal observers are plotted in Figure 2a. Consistent with previous reports ${ }^{21-24}$ where luminance is the same in the two eyes (no ND filter in the current study), the perceived phase of the cyclopean grating gradually shifted from the phase of the left eye (when there was no signal in the right eye, $\delta=0$ ) to be around zero (when the two eyes had equal contrast, $\delta=1$ ), signifying the two eyes are almost functionally balanced. Decreasing mean luminance in the left eye shifted the PvR curve leftward, leading to a displaced balance point and indicating reduced dominance of the left eye relative to the right eye. A repeated-measure within-subject ANOVA revealed that the perceived phase depended strongly on both interocular contrast ratios $(F(5,10)=72.300, p<0.001)$ and mean luminance levels in the left eye $(F(3,6)=68.327, p<0.001)$. The interaction of these two factors was also significant $(F(15,30)=$ 12.258, $p<0.001)$.

To better illustrate the relationship between the interocular luminance difference and the balance point, we re-plotted the contrast

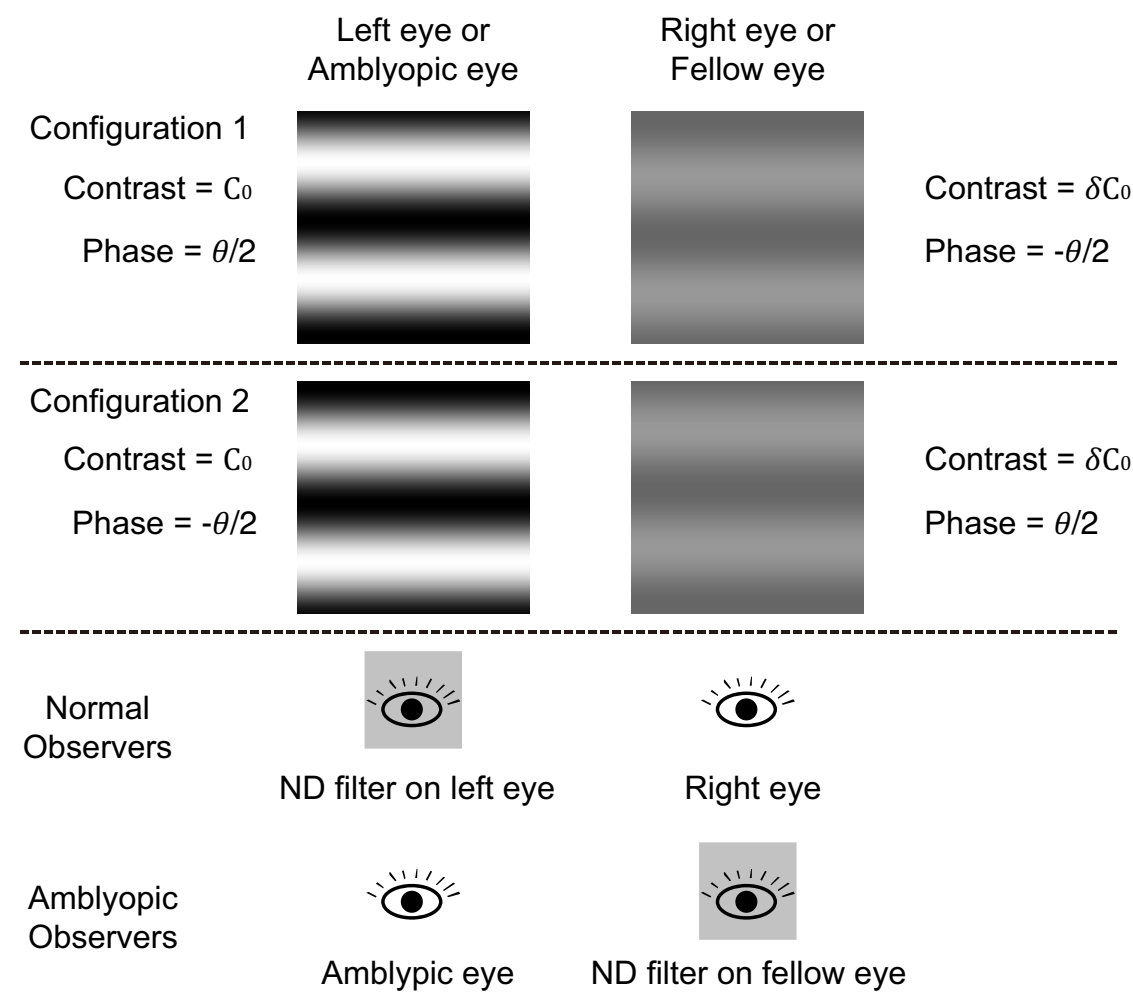

Figure 1 Illustration of the experimental design. Two monocular sine-wave gratings with different contrast and phase (shifted in opposite directions with the same magnitude) were dichoptically presented to two eyes. The contrast of the sine-wave grating in the left eye of normal observers or the amblyopic eye of amblyopes was fixed as ' $C_{0}$ ' (for normal observers, $\mathrm{C}_{0}=64 \%$; for amblyopes, $\mathrm{C}_{0}=100 \%$ ), and the contrast of the sine-wave grating in the other eye was ' $\delta C_{0}$ ' $(\delta=[0,0.1,0.2,0.4,0.8,1.0])$. Two stimulus configurations were used to cancel potential positional biases: in configuration 1 , the phase-shift was ' $\theta / 2=22.5^{\circ}$ ' in the left eye of normal observers (or the amblyopic eye of amblyopes) and ' $-\theta / 2$ ' in the right eye of normal observers (or the fellow eye of amblyopes); in configuration 2 , the phase-shift was ' $-\theta / 2$ ' in the left eye of normal observers (or the amblyopic eye of amblyopes) and ' $\theta / 2$ ' in the right eye of normal observers (or the fellow eye of amblyopes). The perceived phase-shift of the cyclopean sine-wave grating was scored as the difference between the measurements from these two configurations. In the luminance-attenuating measurement, the mean luminance in right eye of normal observers and amblyopic eye of amblyopic observers was fixed as $190 \mathrm{~cd} / \mathrm{m}^{2}$, the mean luminance in the other eye was reduced by using various neutral density (nominal ND) filters whose calibrated densities and associated retinal illuminances were: without ND filter (2908.7 Td), nominal 1ND (calibrated density: 0.999 ND; retinal illuminances: 474.3 Td), nominal 2ND (calibrated density:1.998 ND; retinal illuminances:63.7 Td), and nominal 3ND (calibrated density:3.001 ND; retinal illuminances:7.3 Td). 


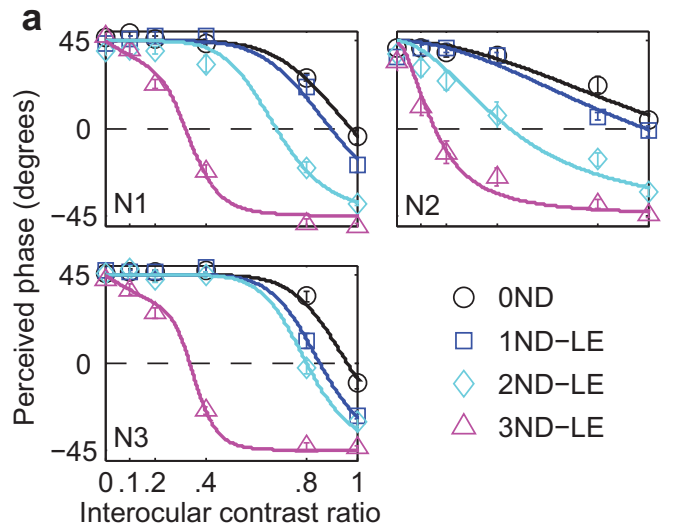

(RE/LE)

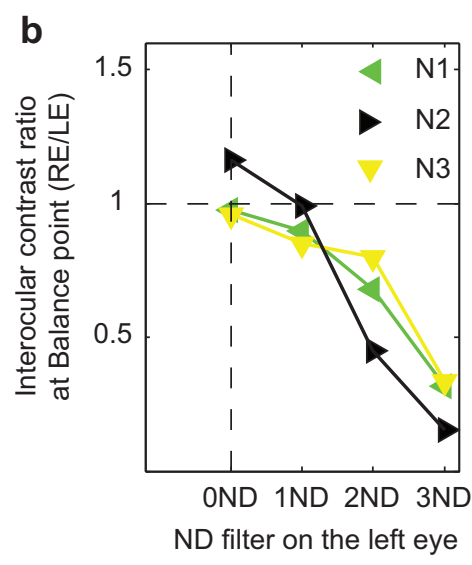

Figure $2 \mid$ The effect of attenuation of left eye's stimulus luminance with neutral density filters on binocular combination in three normal observers. a). The perceived phase versus interocular contrast ratio (PvR) curves at four neutral density transmission rates of the three normal observers. The vertical axis is the perceived phase of the cyclopean sine-wave grating; the horizontal axis is the contrast ratio of the grating in the right eye to the grating in the left eye. The four symbols represent four transmission rates: black ' $\bigcirc$ ' represents no ND filter was added (i.e., 100\% transmission); blue ' $\square$ ' represents 1 ND filter (i.e., $10 \%$ transmission) was added on the left eye; cyan ' $\triangle$ ' represents 2 ND filter (i.e., $1 \%$ transmission) was added on the left eye; purple ' $\triangle$ ' represents $3 \mathrm{ND}$ filter (i.e., $0.1 \%$ transmission) was added on the left eye. The horizontal dashed line in the middle of each panel indicates expected output when the perceived phase is zero. Error bars represent S.E.M. b). The interpolated contrast ratios at balance point versus transmission rates of the three normal observers. The three symbols represent three normal observers: green ' $<$ ', N1; black ' ', N2; yellow ' ', N3. The horizontal black dashed line indicates where the two eyes contribute equally in phase combination. The vertical black dashed line indicates condition where no ND filter was added.

ratios corresponding to the balance points as a function of the neutral density (i.e., transmission levels) in Figure 2b. Averaged over the three observers, the contrast ratio corresponding to the balance points decreased from 1.033 at $190 \mathrm{~cd} / \mathrm{m}^{2}$ (no ND filter; $100 \%$ transmission) to $0.912,0.643$ and 0.269 at 19 (1 ND; $10 \%$ transmission), 1.9 (2ND; $1 \%$ transmission) and 0.19 (3ND, 0.1\% transmission) $\mathrm{cd} / \mathrm{m}^{2}$, respectively. The balance point decreases almost linearly with the neutral density (linear fitting, slope $=-3.723, \mathrm{R}^{2}=0.954$ ). These results indicate that adding an ND filter on the left eye of a normal observer lessens that eye's contribution to the binocular phase combination.

Amblyopic subjects. The measured PvR curves for all the seven amblyopic observers with 2 ND (' $\nabla$ ' symbols) and without any ND filter (' $\bigcirc$ ' symbols) added on the fellow eye are shown in Figure 3. Consistent with previous findings in amblyopia where the two eyes are of equal luminance ${ }^{22,23}$, the perceived phase of the combined image shifted rapidly from the phase of the grating in the
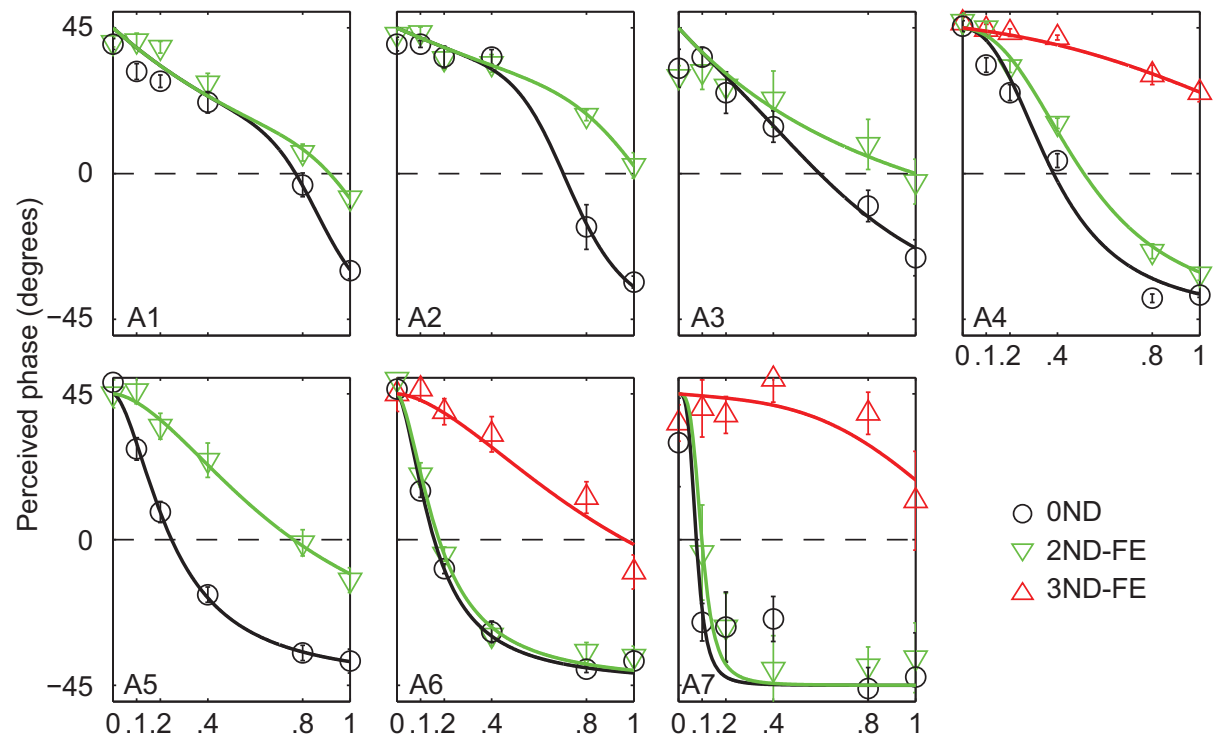

Interocular contrast ratio (FE/AE)

Figure $3 \mid$ The PvR curves without and with ND filters on the fellow eye of seven amblyopic observers. The vertical axis is the perceived phase of the cyclopean sine-wave grating; the horizontal axis is the contrast ratio of the grating in the fellow eye to the grating in the amblyopic eye. The three symbols represent three transmission rates: red ' $\Delta$ ' represents $3 \mathrm{ND}$ filter (i.e., $0.1 \%$ transmission) was added on the fellow eye, i.e., $3 \mathrm{ND}-\mathrm{FE}$; green ' $\nabla$ ' represents $2 \mathrm{ND}$ filter (i.e., $1 \%$ transmission) was added on fellow eye, i.e., $2 \mathrm{ND}$-FE; black ' $\bigcirc$ ’ represents no ND filter was added (i.e., $100 \%$ transmission), i.e., $0 \mathrm{ND}$. The horizontal dashed line in the middle of each panel indicates an expected output when the perceived phase is zero. Error bars represent S.E.M. 


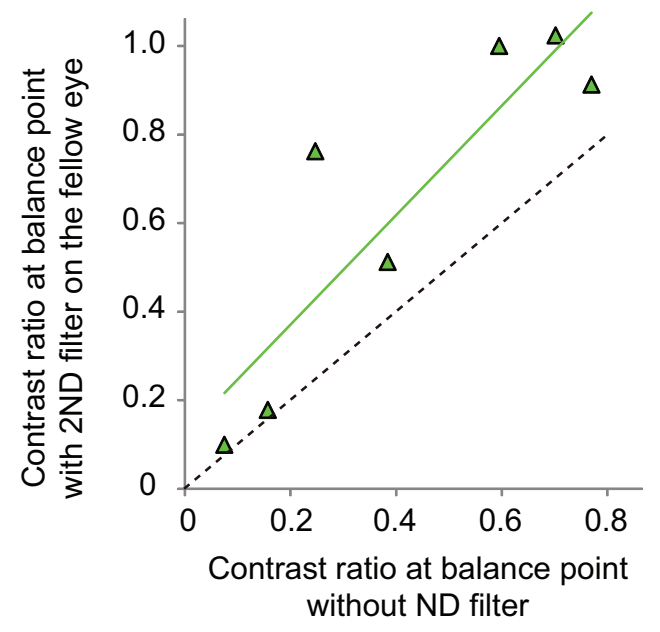

Figure $4 \mid$ The interpolated contrast ratios corresponding to the balance points when a $2 \mathrm{ND}$ filter was placed before the fellow eye versus when no ND filter was used. Results are shown for the seven amblyopes. Each symbol represents one amblyopic observer. The green solid line is the best fitting linear regression line $\left(\mathrm{R}^{2}=0.78\right.$, slope $\left.=1.24\right)$. The black dashed line indicates has a slope of 1 , indicating the prediction of no improvement. Note the fitted curve is not constrained to pass through the origin as zero on the abscissa refers to absence of binocular function (i.e. monocular function) not absence of function altogether.

amblyopic eye ( when $\delta=0$, i.e., blank in the fellow eye), to zero, and to the phase of the grating in the fellow eye (when $\delta=1$, i.e., the two eyes had equal contrast), signifying the fellow eye's dominance over the amblyopic eye. Decreasing mean luminance in the fellow eye with a $2 \mathrm{ND}$ filter displaced the PvR curve rightward, indicating reduced dominance of the fellow eye. A repeated-measure within-subject ANOVA revealed that the perceived phase of the cyclopean grating depended strongly on the interocular contrast ratios $(F(5,30)=32.269, p<0.001)$ and the mean luminance levels $(F(1,6)=18.440, p=0.005)$.

In Figure 4, we plot the interpolated contrast ratios corresponding to the balance points when a $2 \mathrm{ND}$ filter was added on the fellow eye versus that when no ND filter was added for the seven amblyopes. With a $2 \mathrm{ND}$ filter added on the fellow eye, the contrast ratio at the balance point of all the amblyopes improved. This means that placing a 2ND filter in front of the fellow eye ensures a greater degree of balance between the amblyopic and fellow eyes. Averaged across subjects, the contrast ratio at balance point was $0.418 \pm 0.104$ (mean \pm S.E.M) when no ND filter was added (i.e., $100 \%$ transmission) and $0.641 \pm 0.145$ when the luminance was attenuated by a factor of 100 (from 190 to $1.9 \mathrm{~cd} / \mathrm{m}^{2}$ ) in the fellow eye. A one tailed Paired $t$ test showed that the increase was significant: $t(6)=-3.048$, $p=0.011$.

For subjects A4, A6 and A7, their contrast ratios at balance point didn't increase much when the luminance in the fellow eye was reduced by a $2 \mathrm{ND}$ filter (i.e., $1 \%$ transmission). To further verify the effect of decreasing the fellow eye's luminance on the binocular combination for these three amblyopes, their PvR curves were remeasured when a $3 \mathrm{ND}$ filter (i.e., $0.1 \%$ transmission) was added in front of the fellow eye. These results are plotted in Figure 3 using ' $\Delta$ ' symbols. As expected, a further improvement of the contrast ratio at balance point for all these three amblyopes was found when the fellow eye's luminance was decreased further. These results, together with the results of other amblyopes, indicate that the placing of ND filter in front of the fellow eye weakens its contribution to binocular combination.

Modelling. For the phase combination paradigm, Ding and Sperling have developed a gain-control theory to predict the perceived phase of the cyclopean grating in normal vision ${ }^{21}$. According to their theory, images in the two eyes first go through a double interocular contrast gain control pathway and are linear combined thereafter. In the gain-control pathway, each eye exerts gain-control on the other eye's signal in proportion to its own signal contrast-energy, and also exerts gain-control over the other eye's gain-control. The cyclopean image of the left $\left(\operatorname{Lum}_{L}\right)$ and right $\left(\operatorname{Lum}_{R}\right)$ eye inputs was modelled as:

$$
\operatorname{Lum}^{\prime}=\operatorname{Lum}_{L} \times \frac{1}{1+\frac{\varepsilon_{R}}{1+\varepsilon_{L}}}+\operatorname{Lum}_{R} \times \frac{1}{1+\frac{\varepsilon_{L}}{1+\varepsilon_{R}}}
$$

Where $\varepsilon_{L}$ and $\varepsilon_{R}$ are the total contrast energy in the two eyes and are modelled as $\varepsilon_{L}=\rho C_{L}^{\gamma}$ and $\varepsilon_{R}=\rho C_{R}^{\gamma}, \rho$ is the gain-control efficiency of the signal sine-wave grating, and $\gamma$ is the exponent of the non-linear transducer.

The perceived phase difference between the two experimental configurations $\left(\theta^{\prime}\right)$ was then predicted as (see Model derivation in Supplementary online for detailed derivation):

$$
\theta^{\prime}=2 \tan ^{-1}\left[\frac{1-\delta^{1+\gamma}}{1+\delta^{1+\gamma}} \tan \left(\frac{\theta}{2}\right)\right]
$$

According to equation (2), the perceived phase of the cyclopean grating $\left(\theta^{\prime}\right)$ is determined only by one parameter ' $\gamma$ ', and the interocular contrast ratio $(\delta)$ at balance point (i.e., when $\theta^{\prime}=0$ ) will be always at ' 1 ' in normal vision.

Using the same paradigm, Huang, Zhou et al. ${ }^{22,23}$ found stimulus of equal contrast was weighted much less in the amblyopic eye relative to the fellow eye in binocular combination in anisometropic amblyopes. To model this result, they added a multiplicative attenuation factor (or inhibition factor) ' $\eta$ ' to the total contrast energy $(\varepsilon)$ in the amblyopic eye, and predicted the perceived phase of the amblyopic eye $\left(\theta^{\prime}\right)$ as:

$$
\theta^{\prime}=2 \tan ^{-1}\left[\frac{\eta^{1+\gamma}-\delta^{1+\gamma}}{\eta^{1+\gamma}+\delta^{1+\gamma}} \tan \left(\frac{\theta}{2}\right)\right]
$$

According to equation (3), the interocular contrast ratio $(\delta)$ at balance point (i.e., when $\theta^{\prime}=0$ ) will be at ' $\eta$ ' in the amblyopic vision.

In the current study, to quantitatively investigate the role of luminance on the binocular phase combination in both the normal and the amblyopic vision, we assume that the gain-control efficiencies of the signal sine-wave grating in two eyes are different and are affected by the luminance of the input, i.e. $\rho_{L / R} \propto L u m$. Theoretically, a reduction of mean luminance can also affect the transducer non-linearity, i.e. ' $\gamma$ ', in the eye, which is mathematically equivalent to changes in the gain-control efficiency $(\rho)$. However, ' $\gamma$ ' has to increase incredibly high in luminance-attenuated conditions to explain our data, e.g. from 3.948 at $0 \mathrm{ND}$ filter condition increase to 5.351 at $1 \mathrm{ND}, 8.135$ at 2ND and 501.361 at $3 \mathrm{ND}$ on average of three normal subjects. Physiological evidence actually suggests a positive relationship between luminance and non-linearity ${ }^{25}$. We thus hypothesized that luminance changes the gain-control efficiency $(\rho)$.

We then predict the perceived phase difference between the two experimental configurations in normal observers $\left(\theta \_n o r m a l '\right)$ as:

$\theta \_n o r m a l^{\prime}=2 \tan ^{-1}\left[\frac{\left(1+\mu_{N D} \rho_{L} C_{0}^{\gamma}\right)-\left(1+\rho_{R} \delta^{\gamma} C_{0}^{\gamma}\right) \delta}{\left(1+\mu_{N D} \rho_{L} C_{0}^{\gamma}\right)+\left(1+\rho_{R} \delta^{\gamma} C_{0}^{\gamma}\right) \delta} \tan \left(\frac{\theta}{2}\right)\right](4)$

In our modified gain-control model, there are four parameters, $\mu_{N D}$, $\rho_{L}, \rho_{R}$ and $\gamma$. In which, $\rho_{L}$ and $\rho_{R}$ are the gain-control efficiencies of the signal sine-wave grating in the left and the right eye when there is no ND filter, $\mu_{N D} \rho_{L}$ is the gain-control efficiency in the left eye when an ND filter was added on that eye, where $\mu_{N D}$ is a luminance factor on the gain-control efficiency in the left eye $\left(\mu_{N D}=1\right.$ when no ND filter was used). If $\rho_{L}=\rho_{R}$ and $\mu_{N D}=1$, i.e., the two eyes have same gain-control efficiency and luminance, equation (4) will reduce to equation (2). A block diagram of the processes involved in the model 


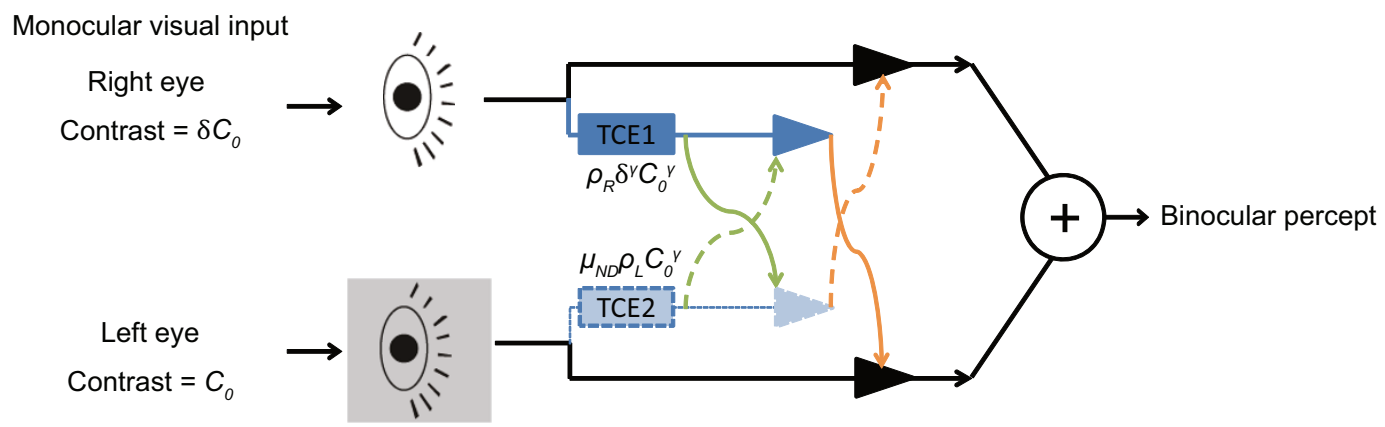

Figure $5 \mid$ A block diagram of the processes involved in the modified gain-control model. Images in the two eyes first go through a double interocular contrast gain control pathway and are linear combined thereafter. In the gain-control pathway, each eye exerts gain-control on the other eye's signal in proportion to its own signal contrast-energy, and also exerts gain-control over the other eye's gain-control. The strength of the gain-control depends on the total contrast energy (TCE, i.e., ' $\varepsilon$ ' in the model derivation) in each eye. The TCE is modelled by $\mu_{N D} \rho_{L} C_{0}^{\gamma}$ in the left eye (the eye with ND filter) and $\rho_{R} \delta^{\gamma} C_{0}^{\gamma}$ in the right eye. In which, $\rho_{L}$ and $\rho_{R}$ are the gain-control efficiencies of the signal sine-wave grating in the left and right eye when there is no ND filter; $\mu_{N D}$ is a luminance factor of the ND filter on the gain-control efficiency in the left eye ( $\mu_{N D}=1$ when no ND filter was used), and $\gamma$ is the exponent of the non-linear transducer.

is provided in Figure 5. For details of the derivation, see Model derivation in Supplementary online.

Similarly for amblyopia, we get:

$\theta \_a m b^{\prime}=2 \tan ^{-1}\left[\frac{\left(1+\rho_{A} C_{0}^{\gamma}\right)-\left(1+\mu_{N D} \rho_{F} \delta^{\gamma} C_{0}^{\gamma}\right) \delta}{\left(1+\rho_{A} C_{0}^{\gamma}\right)+\left(1+\mu_{N D} \rho_{F} \delta^{\gamma} C_{0}^{\gamma}\right) \delta} \tan \left(\frac{\theta}{2}\right)\right]$

In which, $\rho_{A}$ and $\rho_{F}$ are the gain-control efficiencies of the signal sinewave grating in the amblyopic and the fellow eye when there is no ND filter, $\mu_{N D} \rho_{F}$ is the gain-control efficiency in the fellow eye when an ND filter was added on that eye, where $\mu_{N D}$ is a luminance factor on gain-control efficiency in the fellow eye $\left(\mu_{N D}=1\right.$ when no ND filter was used).

The fits of our model to data are plotted as smooth curves in Figures $2 \mathrm{a}$ and 3 . The model successfully accounted for $98.9 \%$ data variance in the normal observers ( 24 data points were fitted with six free parameters) and $96.3 \%$ variance in the amblyopic observers (for subjects A4, A6 and A7, 18 data points were fitted with five free parameters; for other subjects, 12 data points were fitted with four free parameters) on average. Detail model fitting results of normal and amblyopic observers are provided in Tables S2 and S3 in Supplementary online. The fitted luminance factors $\left(\mu_{N D}\right)$ on the gain-control efficiency in the left eye of normal observers $\left(\rho_{L}\right)$ and in the fellow eye of amblyopic observers $\left(\rho_{F}\right)$ at different transmission rates are presented in Table 1 . According to the fitting, the average luminance factors $\left(\mu_{N D}\right)$ on the gain-control efficiency in the left eye $\left(\rho_{L}\right)$ of three normal observers decreased from 1 at $190 \mathrm{~cd} / \mathrm{m}^{2}$ (no ND filter; $100 \%$ transmission) to $0.559,0.154$ and 0.000 at 19 ( $1 \mathrm{ND} ; 10 \%$ transmission), 1.9 (2ND; $1 \%$ transmission) and $0.19\left(3 \mathrm{ND}, 0.1 \%\right.$ transmission) $\mathrm{cd} / \mathrm{m}^{2}$, respectively. While in the seven amblyopic observers, the average luminance factors $\left(\mu_{N D}\right)$ on the gain-control efficiency of the fellow eye $\left(\rho_{F}\right)$ decreased from 1 at $190 \mathrm{~cd} / \mathrm{m}^{2}$ (no ND filter; $100 \%$ transmission) to 0.297 at $1.9 \mathrm{~cd} / \mathrm{m}^{2}$ (2ND; $1 \%$ transmission). There was a trend toward decreasing luminance factors $\left(\mu_{N D}\right)$ on the gain-control efficiency of the fellow eye (i.e., increasing the reduction of $\rho_{F}$ ) being associated with increasing effective contrast ratio at balance point before the adding of ND filter (i.e., decreasing severity of amblyopia), $\mathrm{r}=-0.66, p=$ $0.10,2$-tailed. In other words, the less the sensory imbalance (i.e., less severity of amblyopia), the more the sensitivity to the luminance change of the fellow eye. The corresponding scatter diagram is provided in Fig. S1 in Supplementary.

\section{Discussion}

We demonstrate that the selective reduction of monocular mean luminance in one eye can significantly affect the interocular balance in binocular combination in both normal and amblyopic observers. Normal observers demonstrate an "amblyopic" pattern in as far as binocular phase combination is concerned if the mean luminance in their left eyes is reduced. Amblyopes can also show "normal" behaviour in binocular phase combination if the mean luminance in their fellow eyes is attenuated. Using a modified contrast gain control model, we demonstrate that these phenomena may stem from attenuated gain-control efficiency of the filtered eye. When the left eye's mean luminance in normal observers is reduced by ND filters, the gain-control efficiency of this eye $\left(\rho_{L}\right)$ will be attenuated, thus lowering the contribution of this eye in binocular combination and exhibiting an 'amblyopic-like' behavior. Similarly, attenuation of the fellow eye's mean luminance also attenuates the gain-control efficiency of the eye $\left(\rho_{F}\right)$, thus increasing the contribution of the amblyopic eye in binocular combination and producing 'normallike' behavior in amblyopia.

The modified gain control model that we used in this study can explain the effect of unilateral mean luminance on binocular combination, a contributing to the current information on binocular combination $^{21-24}$. However, the remaining issue is by what mechanism do these gain-control changes occur. Neutral density filters do not change the physical contrast of stimuli, only their mean luminance. The stimulus used here was $0.294 \mathrm{cycle} /{ }^{\circ}$ grating and

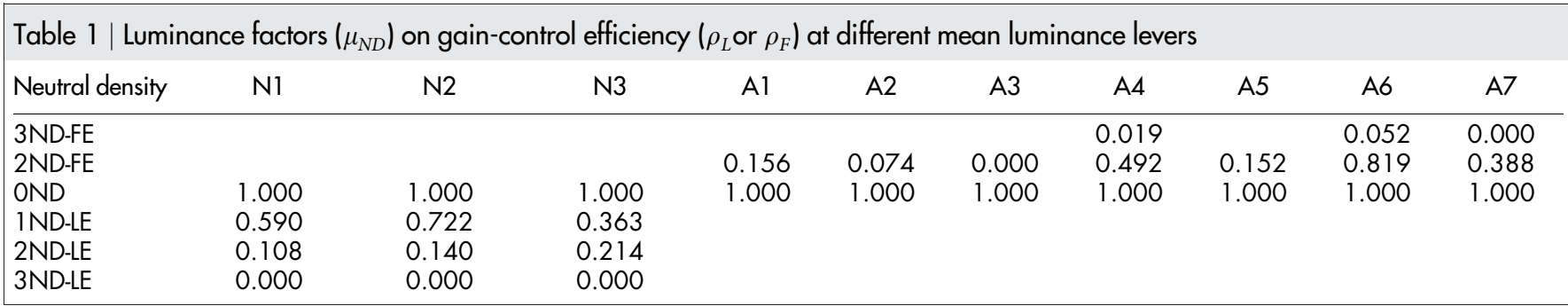


neither its contrast threshold ${ }^{5}$ nor its suprathreshold contrast perception ${ }^{26}$ should have been significantly affected over the range of ND filters (0-3ND) used here. However, there is physiological evidence suggesting that reductions in mean luminance do result in attenuated contrast gain at the single cell level. For example, Purpura and Kaplan et al. ${ }^{27}$ reported such a manipulation reduced the gain of primate $\mathrm{P}$ and $\mathrm{M}$ retinal ganglion cells, $\mathrm{Hess}^{28}$ and Geisler and Albrecht et al. ${ }^{29}$ reported that reductions in mean luminance resulted in reduced contrast gain of simple and complex cells in primary visual cortex of cat.

Our results could have important clinical implications. First of all, amblyopia once thought to be a purely monocular deficit is now increasingly seen as the result of imbalanced binocular interactions, in particular suppression ${ }^{20,22,23,30-39}$. Our manipulation of changing the interocular contribution in binocular combination provides a simple and effective way to modulate interocular balance in amblyopes by reducing the effectiveness of the signal originating from the fellow fixing eye, which might benefit any binocular treatment of amblyopia. Of course balancing the signals from the two eyes for the phase judgment used here may not necessarily result in a balanced contrast ${ }^{23,24}$ and motion ${ }^{32}$ response, we will need to test each of these in turn.

\section{Methods}

Observers. Three normal adults (the first author, JW.Z. and two naive subjects; Age: 27-30 years old) with normal or corrected-to-normal vision and seven randomly chosen adult amblyopes (with strabismus and/or anisometropia; Age: 16-51 years old) with refractive errors corrected participated in this experiment. Detailed characteristics of the amblyopes are listed in Table S1 in Supplementary online. The study was approved by the Institutional Review Board of McGill University and the Institute of Psychology, Chinese Academy of Sciences. Except the first author, all subjects are naive to the purpose of this study. An information consent form was signed by each of them.

Apparatus. The stimuli were generated by a Mac computer (Apple, Cupertino, CA, U.S.A.) running Matlab with PsychToolBox 3.0.9 extensions ${ }^{40,41}$ and dichoptically presented with Z800 dual pro head-mounted goggles (eMagin, Bellevue, WA, U.S.A.), with a refresh rate of $60 \mathrm{~Hz}$ and a resolution of $800 \times 600$. The total view field was 30.26 degrees $\times 22.69$ degrees for each eye. The mean luminance of OLED goggles was $190 \mathrm{~cd} / \mathrm{m}^{2}$. Different mean luminance levels were induced by using various neutral density (nominal ND) filters (Kodak ${ }^{\circledR}$, Rochester, N.Y., U.S.A.) whose calibrated densities and associated retinal illuminances were: without ND filter (2908.7 Td), nominal 1ND (calibrated density: 0.999 ND; retinal illuminances: 474.3 Td), nominal 2ND (calibrated density:1.998 ND; retinal illuminances:63.7 Td), and nominal 3ND (calibrated density:3.001 ND; retinal illuminances:7.3 Td).

Stimuli. Stimuli were two horizontal sine-wave gratings, each subtending $6.81^{\circ} \times$ $6.81^{\circ}$ (i.e. 180 pixels $\times 180$ pixels). These two gratings were dichoptically presented on the centre of eye goggles. A high-contrast frame (width: $0.378^{\circ}$; length: $20.43^{\circ}$ ) with four white diagonal bars (width: $0.378^{\circ}$; length: $9.63^{\circ}$ ) were presented surrounding the grating in each eye to assist observers in maintaining vergence when they perform the tasks.

The luminance profile of the grating on the amblyopic eye of amblyopic observers or the left eye of normal observers is:

$$
\operatorname{Lum}_{A E / L E}(y)=L_{0}\left[1-C_{0} \cos \left(2 \pi y \pm \frac{\theta}{2}\right)\right]
$$

The luminance profile of the grating on the fellow eye of amblyopic observers or the right eye of normal observers is:

$$
\operatorname{Lum}_{F E / R E}(y)=L_{0}\left[1-\delta C_{0} \cos \left(2 \pi y \mp \frac{\theta}{2}\right)\right]
$$

Where $L_{0}$ is the background luminance, $C_{0}$ is the base contrast which is $100 \%$ for amblyopic observers and $64 \%$ for normal observers, $f=0.294 \mathrm{cycle}^{\circ}$ is the spatial frequency of the gratings and $\delta$ is the interocular contrast ratio, $\delta=[0,0.1,0.2,0.4$, $0.8,1.0]$. The two gratings were phase-shifted in opposite directions with same amount of $\theta / 2$ which was $22.5^{\circ}$ in the test.

Procedure. The procedure for measuring phase combination is similar to that described in our previous publications ${ }^{22-24}$. Observers fulfilled two consecutive tasks in each trial. In the first task, i.e. the alignment task, a fixation display consisting of binocular fixation crosses $\left(3.78^{\circ} \times 3.78^{\circ}\right)$ and four monocular dots $\left(0.378^{\circ}\right.$ in diameter), of which two were in the $1^{\text {st }}$ and $3^{\text {rd }}$ quadrants in the left eye and two in the $2^{\text {nd }}$ and $4^{\text {th }}$ quadrants in the right eye, were presented in the centre of the larger highcontrast frame together with four white diagonal lines. Observers were instructed to move the image in their amblyopic eye (or left eye in normal observers) using up, down, left and right arrow keys to fuse the images from two eyes. After achieving stable vergence, observers were asked to press the 'space' key. This followed by a $500 \mathrm{~ms}$ presentation of the frames, and then the second task, i.e. phase adjustment task, in which the two sine-wave gratings were presented in the centre. Observers were asked to adjust the height of a 1-pixel sided reference line to indicate the perceived phase of the cyclopean sine-wave grating after binocular combination, defined as the location of the centre of the dark stripe of the grating. The initial height of the reference line was randomly $([-9,10]$ pixels) positioned relative to the centre of the frame in each trial. The reference line was moved with a fixed step size of one pixel, corresponding to $4^{\circ}$ phase angle of the sine-wave grating. Subjects were asked to press the 'space' key again to register their reports, which also initiated the next trial that was preceded by a 1-s inter-trial interval. A typical trial lasted about eight seconds.

Design. We measured the perceived phase of the cyclopean sine-wave grating as a function of the contrast ratio between input image in the right eye to that of the image in the left eye in normal observers and between input of the fellow eye to that of the input of the amblyopic eye in amblyopic observers at different mean luminance levels. The following types of interocular contrast ratios were used: $0,0.1,0.2,0.4,0.8$ and 1 The interocular phase difference was fixed at $45^{\circ}$ to minimize potential rivalry $y^{24,42}$. Two stimulus configurations were used to cancel potential positional biases ${ }^{21-24}$ : in configuration 1, the phase-shift was $22.5^{\circ}$ in the left eye of normal observers (or the amblyopic eye of amblyopes) and $-22.5^{\circ}$ in the right eye of normal observers (or the fellow eye of amblyopes); in configuration 2 , the phase-shift was $-22.5^{\circ}$ in the left eye of normal observers (or the amblyopic eye of amblyopes) and $22.5^{\circ}$ in the right eye of normal observers (or the fellow eye of amblyopes). The perceived phase of the cyclopean sine-wave grating was scored as the difference between the measurements from these two configurations. There were, therefore, a total of 12 conditions (6 interocular contrast ratios $\times 2$ configurations) in one measure of the perceived phase versus interocular contrast ratio curve (PvR curve).

For normal observers, PvR curves were measured at four different transmission rates in the left eye: $100 \%$ (no ND filter), $10 \%$ (1ND), $1 \%$ (2ND) and $0.1 \%$ (3ND). For amblyopic observers, PvR curves were measured when a $2 \mathrm{ND}$ filter was added in front of the fellow eye and when no ND filter was used. Luminance in the right eye of normal observers and the amblyopic eye of amblyopes was not attenuated. For subjects A4, A6 and A7, their PvR didn't change much when the luminance in the fellow eye was reduced by a $2 \mathrm{ND}$ filter. To further verify the effect of decreasing the fellow eye's luminance on the binocular combination for these three amblyopes, their PvR curves were re-measured using a $3 \mathrm{ND}$ filter in front of the fellow eye. Three minutes of adaptation were allowed before the measurement in each condition. For PvR curves at each transmission rate, the measurement was repeated eight times in separate blocks. Observers could take an optional rest in the measurement. Demos of the task and practice trials were provided prior to data collection.

Data fitting procedure. All the model-fitting procedures were implemented in Matlab using a non-linear least-square method and weighted by the stand error of each data point. The goodness-of-fit was evaluated by the $r^{2}$ statistic:

$$
r^{2}=1-\frac{\sum\left(y_{i}^{\text {predicted }}-y_{i}^{\text {measured }}\right)^{2}}{\sum\left[y_{i}^{\text {measured }}-\text { mean }\left(y_{i}^{\text {measured }}\right)\right]^{2}}
$$

1. Chang, Y. H. et al. The effects of interocular differences in retinal illuminance on vision and binocularity. Graefes Arch Clin Exp Ophthalmol 244, 1083-1088 (2006).

2. von Noorden, G. K. \& Burian, H. M. Visual acuity in normal and amblyopic patients under reduced illumination: I. Behavior of visual acuity with and without neutral density filter. Archives of Ophthalmology 61, 533 (1959).

3. De Valois, R. L., Morgan, H. \& Snodderly, D. M. Psychophysical studies of monkey vision-III. Spatial luminance contrast sensitivity tests of macaque and human observers. Vision Res 14, 75-81 (1974).

4. Owsley, C., Sekuler, R. \& Siemsen, D. Contrast sensitivity throughout adulthood. Vision Res 23, 689-699 (1983).

5. Hess, R. F. \& Howell, E. R. Detection of low spatial frequencies:A single filter or multiple filters? Ophthal. Physiol. Opt. 8, 378-385 (1988).

6. Van Nes, F. L. \& Bouman, M. A. Spatial modulation transfer in the human eye. J. Opt. Soc. Am. 57, 401-406 (1967).

7. van Nes, F. L., Koenderink, J. J., Nas, H. \& Bouman, M. A. Spatiotemporal modulation transfer in the human eye. J Opt Soc Am 57, 1082-1088 (1967).

8. Johnson, C. A. Effects of luminance and stimulus distance on accommodation and visual resolution. Journal of the Optical Society of America 66, 138-142 (1976).

9. Banks, M. S., Geisler, W. S. \& Bennett, P. J. The physical limits of grating visibility. Vision Res 27, 1915-1924 (1987).

10. Shapley, R. \& Victor, J. The contrast gain control of the cat retina. Vision Res 19 , 431-434 (1979)

11. Lovasik, J. V. \& Szymkiw, M. Effects of aniseikonia, anisometropia, accommodation, retinal illuminance, and pupil size on stereopsis. Invest Ophthalmol Vis Sci 26, 741-750 (1985).

12. Gilchrist, J. \& McIver, C. Fechner's paradox in binocular contrast sensitivity. Vision Res 25, 609-613 (1985).

13. Gilchrist, J. \& Pardhan, S. Binocular contrast detection with unequal monocular illuminance. Ophthalmic and physiological optics 7, 373-377 (1987). 
14. Baker, D. H., Meese, T. S., Mansouri, B. \& Hess, R. F. Binocular summation of contrast remains intact in strabismic amblyopia. Invest Ophthalmol Vis Sci 48, 5332-5338 (2007)

15. Verhoeff, F. H. Effect on stereopsis produced by disparate retinal images of different luminosities. Arch Ophthal. 10, 640-645 (1933).

16. Leonards, U. \& Sireteanu, R. Interocular suppression in normal and amblyopic subjects: the effect of unilateral attenuation with neutral density filters. Attention, Perception, \& Psychophysics 54, 65-74 (1993).

17. Trick, G., Dawson, W. \& Compton, J. Interocular luminance differences and the binocular pattern-reversal visual-evoked response. Invest Ophthalmol Vis Sci 22, 394-401 (1982).

18. Heravian-Shandiz, J., Douthwaite, W. \& Jenkins, T. Binocular interaction with neutral density filters as measured by the visual evoked response. Optometry and vision science: official publication of the American Academy of Optometry 68, 801 (1991).

19. Zhang, P., Bobier, W., Thompson, B. \& Hess, R. F. Binocular Balance in Normal Vision and Its Modulation by Mean Luminance. Optometry \& Vision Science 88, 1072 (2011).

20. Hess, R. F., Mansouri, B., Thompson, B. \& Gheorghiu, E. Latent stereopsis for motion in depth in strabismic amblyopia. Invest Ophthalmol Vis Sci 50, 50065016 (2009).

21. Ding, J. \& Sperling, G. A gain-control theory of binocular combination. Proc Natl Acad Sci U S A 103, 1141-1146 (2006).

22. Huang, C. B., Zhou, J. W., Lu, Z. L., Feng, L. X. \& Zhou, Y. F. Binocular combination in anisometropic amblyopia. J Vis 9, 17, 11-16 (2009).

23. Huang, C. B., Zhou, J. W., Lu, Z. L. \& Zhou, Y. F. Deficient binocular combination reveals mechanisms of anisometropic amblyopia: Signal attenuation and interocular inhibition. J Vis 11, 4, 1-17 (2011).

24. Huang, C. B., Zhou, J., Zhou, Y. \& Lu, Z. L. Contrast and phase combination in binocular vision. PLoS One 5, e15075 (2010a)

25. Linsenmeier, R. A. \& Jakiela, H. G. Non-linear spatial summation in cat retinal ganglion cells at different background levels. Exp Brain Res 36, 301-309 (1979).

26. Hess, R. F. Vision at low light levels: role of spatial, temporal and contrast filters. Ophthal. Physiol. Opt. 10, 351-359 (1990b).

27. Purpura, K., Kaplan, E. \& Shapley, R. M. Background light and the contrast gain of primate $\mathrm{P}$ and $\mathrm{M}$ retinal ganglion cells. Proc Natl Acad Sci U S A 85, 4534-4537 (1988).

28. Hess, R. F. The Ed ridge - Green Lecture Vision at low light levels: role of spatial, temporal and contrast filters. Ophthalmic and physiological optics 10, 351-359 (1990a

29. Geisler, W. S., Albrecht, D. G. \& Crane, A. M. Responses of neurons in primary visual cortex to transient changes in local contrast and luminance. The Journal of neuroscience 27, 5063-5067 (2007).

30. Thompson, B. et al. Impaired spatial and binocular summation for motion direction discrimination in strabismic amblyopia. Vision Res (2011).

31. Huang, C. B., Zhou, J., Zhou, Y. \& Lu, Z. L. Deficient Binocular Combination Reveals Mechanisms of Anisometropic Amblyopia. J Vis 10, 466-466 (2010b)

32. Mansouri, B., Thompson, B. \& Hess, R. F. Measurement of suprathreshold binocular interactions in amblyopia. Vision Res 48, 2775-2784 (2008).

33. Baker, D. H., Meese, T. S. \& Hess, R. F. Contrast masking in strabismic amblyopia: attenuation, noise, interocular suppression and binocular summation. Vision Res 48, 1625-1640 (2008).
34. Narasimhan, S., Harrison, E. \& Giaschi, D. Quantitative measurement of interocular suppression in children with amblyopia. J Vis 11, 410-410 (2011)

35. Chen, V. J. \& Tarczy-Hornoch, K. Functional magnetic resonance imaging of binocular interactions in visual cortex in strabismus. Journal of pediatric ophthalmology and strabismus 48, 366 (2011).

36. Li, J. et al. The role of suppression in amblyopia. Invest Ophthalmol Vis Sci 52, 4169-4176 (2011).

37. Thompson, B., Mansouri, B., Koski, L. \& Hess, R. F. From motor cortex to visual cortex: The application of noninvasive brain stimulation to amblyopia. Developmental Psychobiology (2010).

38. Maehara, G., Thompson, B., Mansouri, B., Farivar, R. \& Hess, R. F. The perceptual consequences of interocular suppression in amblyopia. Invest Ophthalmol Vis Sci 52, 9011-9017 (2011)

39. Huang, C. B., Zhou, J., Zhou, Y. \& Lu, Z. L. Temporal Dynamics of Binocular Rivalry in Normal and Amblyopic Vision. J Vis 11, 406-406 (2011).

40. Brainard, D. H. The Psychophysics Toolbox. Spatial Vision 10, 433-436 (1997)

41. Pelli, D. G. The VideoToolbox software for visual psychophysics: Transforming numbers into movies. Spatial Vision 10, 437-442 (1997).

42. Baker, D. H., Wallis, S. A., Georgeson, M. A. \& Meese, T. S. The Effect of Interocular Phase Difference on Perceived Contrast. PLoS One 7, e34696 (2012).

\section{Acknowledgements}

This work was supported by a CIHR grant (\#53346 and 10818) to RFH; the Knowledge Innovation Program of the Chinese Academy of Sciences, and Institute of Psychology (Grant No. Y1CX201006) to CBH. We gratefully acknowledge fruitful and insightful e-mail discussions with Dr. Kurt Simons on the relationship between mean light level and suppression.

\section{Author Contributions}

J.-W.Z., C.-B.H. and R.-F.H. conceived the experiments. J.-W.Z. and W.-L. J. performed the experiments. J.-W.Z., C.-B.H. and R.-F.H. analyzed the data, interpreted the data and wrote the manuscript. All authors reviewed the manuscript.

\section{Additional information}

Supplementary information accompanies this paper at http://www.nature.com/ scientificreports

Competing financial interests: The authors declare no competing financial interests.

How to cite this article: Zhou, J., Jia, W., Huang, C.-B. \& Hess, R.F. The Effect of Unilateral Mean Luminance on Binocular Combination in normal and amblyopic vision. Sci. Rep. 3, 2012; DOI:10.1038/srep02012 (2013)

This work is licensed under a Creative Commons AttributionNonCommercial-ShareAlike 3.0 Unported license. To view a copy of this license, visit http://creativecommons.org/licenses/by-nc-sa/3.0 